EDITORIAL

\title{
The MADIT II and COMPANION studies: will they affect uptake of device treatment?
}

\section{J M Morgan}

Primary prophylaxis of sudden cardiac death by implantable cardioverter-defibrillator (ICD) treatment will greatly increase ICD implant numbers. This will have major cost and infrastructure consequences. Those studies that have demonstrated the clinical need have been industry driven. Whether their conclusions should now expand ICD indications is debated, but it would be perverse to suggest that hesitancy in ICD treatment expansion relates to reservation about the clinical science rather than to concern about cost and resource implications

Correspondence to: Dr John M Morgan, Wessex Cardiothoracic Centre, Southampton University Hospitals, Tremona Road, Southampton SO16 6YD, UK; jmm@cardiology.co.uk t is accepted that sudden cardiac death, often caused by ventricular arrhythmia, is a major cause of western population mortality. ${ }^{1-2}$ Immediate defibrillation is the only remedy for arrhythmic sudden death caused by haemodynamically compromising ventricular tachycardia and ventricular fibrillation, ${ }^{3}$ although pace termination of ventricular tachycardia may prevent the arrhythmic cascade to ventricular fibrillation. ${ }^{4-6}$ Immediate defibrillation shock treatment delivery by the implantable cardioverter-defibrillator (ICD) is highly efficacious in preventing sudden cardiac death. ${ }^{78}$ Though the ICD has been in clinical use for 23 years, ${ }^{9}$ indications for its use have broadened in the 1990s as clinical acceptability of ICD treatment has increased (with the advent of small devices capable of pectoral implantation, using per venous defibrillation leads). ${ }^{10}{ }^{11}$

\section{IMPLANTATION GUIDELINES}

Implantation guidelines based on outcomes of well designed studies have become widely accepted..$^{12}$ These have mainly defined implantation practice in patients who have already suffered ventricular arrhythmia from which they have been rescued-that is, "secondary prevention" of sudden cardiac death. However, the burden of sudden cardiac death mortality rests with those patients who die at the time that they suffer their first ventricular arrhythmia. ${ }^{14}$ Thus the greater challenge has been identification and treatment of these patients before their arrhythmic catastrophe.

Studies of risk factors for sudden cardiac death have allowed risk stratification of such patients and their effective treatment requires implantation of an ICD. ${ }^{715}{ }^{16}$ However, acceptance of this risk stratification and translation into acceptance of the use of ICDs for primary prophylaxis of sudden cardiac death is debated. Furthermore,
Heart 2004;90:243-245. doi: 10.1136/hrt.2002.007807

over the past decade cardiac resynchronisation therapy (CRT) has been developed as a treatment for optimisation of ventricular function in patients with heart failure and impaired ventricular function. ${ }^{17}{ }^{18}$ There is a population overlap between those patients suffering the clinical syndrome termed heart failure and those stratified to be at risk of sudden cardiac death as a consequence of impaired left ventricular function-itself the most important determinant of prognosis and accurate marker for sudden cardiac death. Therefore the debate about ICD implantation in patients with severely impaired left ventricular function must be widened to include management of heart failure by cardiac resynchronisation device therapy and the impact of that on patient prognosis and wellbeing.

\section{STUDIES OF SUDDEN CARDIAC DEATH PRIMARY PROPHYLAXIS}

The goal of the original MADIT study, ${ }^{7}$ which despite criticism has seen its wisdom incorporated into international guidelines, ${ }^{12}{ }^{13}$ was to identify patients at risk of sudden cardiac death in whom that risk was sufficiently great to warrant implantation of an ICD. Key to this study was severe impairment of left ventricular function (ejection fraction $<35 \%$ ) with identification of ventricular arrhythmia on ambulatory monitoring and inducibility of ventricular arrhythmia despite antiarrhythmic drug treatment. There were weaknesses in that study due to the backdrop of rapidly evolving ICD technological change, a therapy cascade that emphasised North American clinical practice and the lack of uptake of $\beta$ blockade by patients in the study. Also, by recruiting patients who remained inducible for ventricular arrhythmias at electrophysiological study despite antiarrhythmic drug treatment, it could be argued that a higher risk population was selected which was more likely to show a benefit from ICD implantation. Nevertheless MADIT outcome data were supported by the data published in the MUSTT study ${ }^{15}$ which, though not a study of ICD treatment itself, identified ICD treatment as the only therapeutic strategy to offer mortality

Abbreviations: CARE-HF, cardiac resynchronisation in heart failure; COMPANION, comparison of medical therapy, pacing and defibrillation in heart failure; CRT, cardiac resynchronisation therapy; ICD, implantable cardioverter-defibrillator; MADIT, multi-center autonomic defibrillator implantation trial; MUSTT, multicenter unsustained tachycardia trial 
reduction in a study population similar to that recruited to MADIT.

To answer the criticisms and expand the reach of primary prophylaxis, the MADIT II study was designed and conducted, and reported in 2002. ${ }^{16}$ Principally risk stratification was by severe left ventricular dysfunction (gauged as an ejection fraction less than $30 \%$ in the context of coronary artery disease) but remote from acute myocardial infarction. Randomisation in a 3:2 fashion (device to optimised medical treatment (for improvement of left ventricular function) limbs) resulted in a significant reduction in mortality (hazard ratio $0.69 ; \mathrm{p}<0.016)$ in the ICD treated group. The statistical design tested all cause mortality as a primary end point and this was reached with an average follow up of only 20 months. Thus the study was terminated with short patient follow up relative to the longevity expected of the implanted devices. Therefore, it can only be inferred that mortality benefit conferred by ICD treatment is maintained over the lifetime of an implanted ICD. This has important consequences for cost efficacy analysis.

Implantation of a device commits the healthcare purchaser to patient treatment at least for the lifetime of an ICD, even if the clinical value of ICD treatment declines with longer observation periods. "Premature achievement" of the study end point may have exaggerated the benefits of ICD treatment. Although the study has served to bolster the validity of MADIT/MUSTT outcomes, the suspicion remains that the longevity benefit of "primary prophylaxis" ICD treatment is exaggerated. ${ }^{19}$ Cost efficacy assessments also challenge widespread adoption of primary prophylaxis in this patient population. Adoption of ICD treatment for the MADIT II patient indication might lead to an enormous increase in ICD implantation rate..$^{20}$ Simplistic cost efficacy calculations indicate that 11 patients would need to receive device treatment to save one life. ${ }^{21}$ It is likely that this represents an exaggeration of costs over benefit, but with the variability of drivers in cost efficacy analyses, robust argument is required to demonstrate that widespread uptake of ICD treatment is warranted in all patients with severely impaired ventricular function. A subgroup analysis has suggested that maximum prognostic benefit is conferred by selecting out those patients with an intraventricular conduction defect (QRS duration > $120 \mathrm{~ms}$ ), which may act as a marker for enhanced risk of sudden cardiac death. ${ }^{22}$ That the hazard ratio is strongly weighted in favour of ICD treatment in such patients does not negate the fact that the hazard ratio is only less strongly, not weakly, weighted in favour of ICD treatment in patients with severely impaired ventricular function but without QRS prolongation. Thus any rationing by healthcare purchasers to this subpopulation would be based on economic rather than clinical considerations.

However, identification of this "higher risk" population adds a further complexity to the argument. There is growing evidence that patients with prolonged intraventricular conduction and dysynchrony of left ventricular contraction may benefit from CRT. To date CRT benefit has been studied in patients with moderate to severe clinical heart failure. ${ }^{17} 1823$ There are no data that determines whether use of CRT before development of heart failure is of benefit in patients who have severe impairment of left ventricular function and in whom, given the MADIT II study, ICD treatment is mandated to prevent sudden cardiac death.

\section{ICD TREATMENT IN HEART FAILURE}

A range of studies has investigated the clinical benefit of CRT in patients with severe impairment of left ventricular function and the clinical syndrome of heart failure. ${ }^{17} 1823$ However, mortality benefit has not been a feature of those studies. It was hoped that the COMPANION study would clarify the impact of CRT on mortality. An end point of mortality and all cause hospitalisation was used to reflect the study designs customarily adopted in pharmacotherapy studies. Nevertheless it was the expectation of the electrophysiological community that this study would clarify the mortality benefit conferred by CRT. COMPANION showed that a significant reduction in the primary end point occurred for both CRT alone and CRT-ICD patients, although it was only in the CRT-ICD treatment group that mortality was significantly reduced (by $40 \%$ from $19 \%$ to $11 \%$ ). Again there was "premature" termination of an implantable device study predicated on a combined end point for which the event rate was greater than had been anticipated. ${ }^{24}$ This led to termination of the study before a reduction in mortality caused by RCT alone could be demonstrated. On ethical grounds there has been crossover of all CRT alone patients to the combined CRT-ICD limb, and as a consequence we will not know from this study with statistical certainty whether, with longer follow up, patient survival is improved by CRT alone. CARE-HF and SCD-HeFT may shed further light on the issue. ${ }^{25} 26$

Subgroup analysis has raised further important issues. For the first time, prophylactic ICD implantation in patients with severely impaired left ventricular function as a consequence of idiopathic dilated cardiomyopathy has been shown. Indeed the mortality benefit from ICD implantation in this population group was greater than that for patients in whom coronary artery disease was the mechanism of left ventricular dysfunction. This should not surprise us given the demonstrable high risk of sudden death for patients with impaired left ventricular function in studies assessing the impact of pharmacotherapies in such patients (including those in whom symptomatic heart failure is well controlled). Despite presentation of the study data, it is discomfiting that the full study and its cost efficacy data have yet to be published. Nevertheless, that CRT combined with primary prophylaxis of sudden cardiac death (ICD treatment) is optimal therapy in patients with severely impaired ventricular function and heart failure is the emerging conclusion. Whether patients in the MADIT II population who have yet to develop clinical heart failure should be considered for prophylactic CRT, with or even without evidence of ventricular dysynchrony, is unknown

\section{CONCLUSION}

Have these studies proven the clinical efficacy of device treatment in prolonging life in patients with severely impaired ventricular function? The level of proof is limited by study design. However, both on practical and ethical grounds these studies are unrepeatable. For the scientific community to criticise industry driven studies when there is neither the will nor the finance within that community to conduct studies on this scale is perverse. It is disingenuous to suggest that the evidence is not compelling. It is an inevitability that ICD treatment uptake will increase in the UK. It may be that the simplicity of risk stratification will obviate an increased infrastructure for electrophysiological assessment but will require an increase in the infrastructure to support device management. The further expansion of CRT that will accompany ICD treatment expansion will require greater sophistication of the follow up approach.

\section{REFERENCES}

1 Jimenez RA, Myerburg RJ. Sudden cardiac death. Magnitude of the problem, substrate/trigger interaction, and populations at high risk. Cardiol Clin 1993;11:1-9.

2 Priori SG, Borggrefe M, Camm AJ, et al. Unexplained cardiac arrest. The need for a prospective registry. Eur Heart J 1992;13:1445-6.

3 Alexander S. Conversion of cardiac arrhythmias by electrical countershock: method, results and indications. Lahey Clin Found Bull 1965; 14:49-57. 
4 Friedberg CK, Lyon $\amalg$, Donoso E. Suppression of refractory recurrent ventricular tachycardia by transvenous rapid cardiac pacing and antiarrhythmic drugs. Report of seven cases. Am Heart J 1970;79:44-50.

5 Wietholt $D$, Block $M$, Isbruch $F$, et al. Clinical experience with antitachycardia pacing and improved detection algorithms in a new implantable cardioverterdefibrillator. J Am Coll Cardiol 1993;21:885-94

6 Bonnet CA, Fogoros RN, Elson JJ, et al. Long-term efficacy of an antitachycardia pacemaker and implantable defibrillator combination. Pacing Clin Electrophysiol 1991;14(5 Pt 1):814-22.

7 Moss AJ, Hall WJ, Cannom DS, et al. Improved survival with an implanted defibrillator in patients with coronary disease at high risk for ventricular arrhythmia. N Engl J Med 1996;335:1933-40.

8 The Antiarrhythmics versus Implantable Defibrillators (AVID) Investigators. A comparison of antiarrhythmic-drug therapy with implantable defibrillators in patients resuscitated from near-fatal ventricular arrhythmias. N Engl J Med 1997:337:1576-84.

9 Mirowski M, Reid PR, Mower MM, et al. Termination of malignant ventricular arrhythmias with an implanted automatic defibrillator in human beings. N Engl J Med 1980;303:322-4.

10 Bardy GH, Hofer B, Johnson G, et al. Implantable transvenous cardioverterdefibrillators. Circulation 1993;87: 1152-68.

11 Yee R, Klein GJ, Leitch JW, et al. A permanent transvenous lead system for an implantable pacemaker cardioverter-defibrillator. Nonthoracotomy approach to implantation. Circulation 1992:85:196-204.

12 American College of Cardiology/American Heart Association Task Force on Practice Guidelines. ACC/AHA/NASPE 2002 guideline update for implantation of cardiac pacemakers and antiarrhythmia devices, 2002.

13 National Institute for Clinical Excellence. Guidance on the use of implantable cardioverter defibrilllator for arrhythmias. www.nice.org Technology appraisal guidance number 11, 2000.

14 Cleland JG, Chattopadhyay S, Khand A, et al. Prevalence and incidence of arrhythmias and sudden death in heart failure. Heart Fail Rev 2002;7:229-42.
15 Buxton $\mathrm{AE}$, Lee $\mathrm{KL}$, Fisher JD, et al. A randomized study of the prevention of sudden death in patients with coronary artery disease. Multicenter unsustained tachycardia trial investigators. N Engl J Med 1999;341:1882-90.

16 Moss AJ, Zareba W, Hall WJ, et al. Prophylactic implantation of a defibrillator in patients with myocardial infarction and reduced ejection fraction. N Engl J Med 2002;346:877-83.

17 Abraham WT, Fisher WG, Smith AL, et al. Cardiac resynchronization in chronic heart failure. N Engl J Med 2002;346:1845-53.

18 Cazeau S, Leclercq C, Lavergne T, et al. Effects of multisite biventricular pacing in patients with heart failure and intraventricular conduction delay. N Engl J Med 2001;344:873-80.

19 Coats AJ. MADIT II, the multi-center autonomic defibrillator implantation trial II stopped early for mortality reduction: has ICD therapy earned its evidencebased credentials? Int J Cardiol 2002;82:1-5.

20 Plummer CJ, Irving RJ, McComb JM. Implications of national guidance for implantable cardioverter defibrillation implantation in the United Kingdom. Pacing Clin Electrophysiol 2003;26(1 Pt 2):479-82.

21 Moss AJ. MADIT-II and its implications. Eur Heart J 2003;24:16-18.

22 luliano S, Fisher SG, Karasik PE, et al. QRS duration and mortality in patients with congestive heart failure. Am Heart J 2002;143:1085-91.

23 Auricchio A, Stellbrink C, Block M, et al. Effect of patients with congestive heart failure. The pacing therapies for congestive heart failure study group. The Guidant congestive heart failure research group. Circulation 1999.99.2993-3001.

24 Salukhe TV, Francis DP, Sutton R. Comparison of medical therapy, pacing and defibrillation in heart failure (COMPANION) trial terminated early; combined biventricular pacemaker-defibrillators reduce all-cause mortality and hospitalization. Int J Cardiol 2003:87:119-20.

25 Cleland JG, Daubert JC, Erdmann E, ef al. The CARE-HF study (cardiac resynchronisation in heart failure study): rationale, design and end-points. Eur J Heart Fail 2001;3:481-9.

26 Klein $\mathrm{H}$, Aurrichio A, Reek $\mathrm{S}$, et al. New primary prevention trials of sudden cardiac death in patients with left ventricular dysfunction: SCD-HEFT and MADIT-II. Am J Cardiol 1999;83(suppl D):91D-7D.

\section{IMAGES IN CARDIOLOGY}

\section{Balloon dilation of the stenotic fifth aortic arch in a newborn with double lumen aortic arch}

$\wedge$ male infant was referred to us at 11 days of life because of cardiac murmur and tachypnoea. Echocardiography at presentation and cardiac catheterisation at 20 days of life revealed a right sided double lumen aortic arch with hypoplastic fourth arch and stenotic fifth arch (left panel), aberrant retro-oesophageal innominate artery (middle panel) with a remnant of a left arterial duct (white arrowhead), and ventricular septal defect. The diameter of the fourth arch was $5.0 \mathrm{~mm}$ and the origin of the fifth arch was $6.4 \mathrm{~mm}$, but the diameter of stenosis of

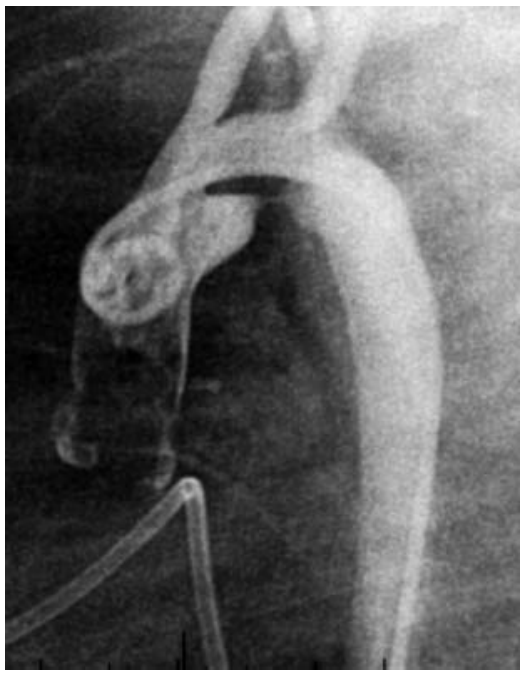

the fifth arch was only $1.2 \mathrm{~mm}$ with a string-like blood flow through it. The infant showed coarctation physiology with a pressure gradient of $28 \mathrm{~mm} \mathrm{Hg}$ between the ascending and descending aorta. Because the infant continued to show signs of congestive heart failure, he underwent successful balloon dilation of the stenotic fifth aortic arch at 32 days old. Retrogradely we dilated the stenosis repeatedly using two different sized balloons, $3 \mathrm{~mm}$ and $5 \mathrm{~mm}$ in diameter, respectively (Cordis, Miami Lakes, Florida, USA). After these procedures, the diameter of stenosis increased

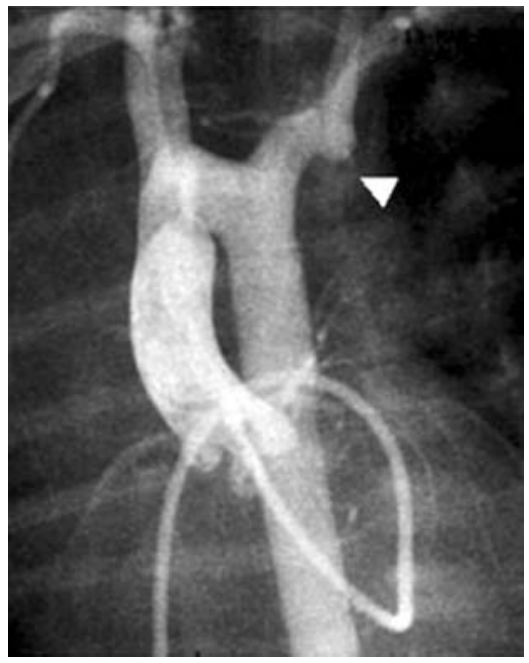

to $4.1 \mathrm{~mm}$ and the pressure gradient disappeared (right panel). The patient was discharged three days after these procedures and has been doing well without any sign of decompensation. This case shows that balloon dilation of the fifth aortic arch is a safe and effective alternative to surgery as a treatment for double lumen aortic arch with hypoplastic fourth aortic arch and stenotic fifth aortic arch in infants.

K Suda

M Matsumura

M Matsumoto kensuda@tenriyorozu-hp.or.jp

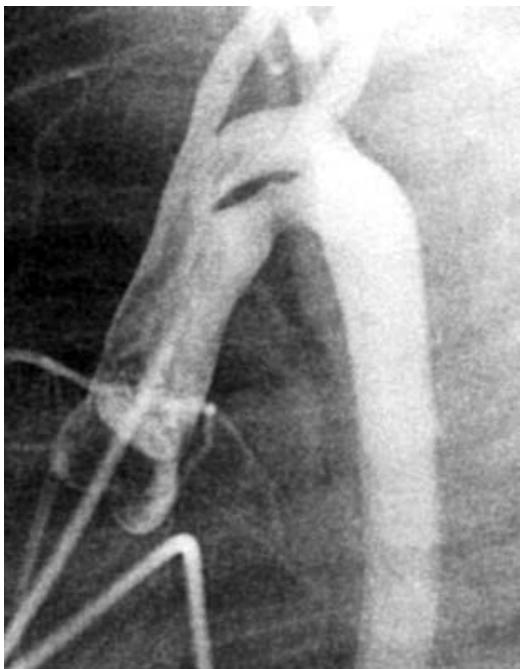

